\title{
RECQ helicase, missense mutations, oxidative stress and BLM gene defect in bloom syndrome
}

\begin{abstract}
Bloom syndrome is a pleiotropic genetic disorder that belongs to group of chromosomal breakage syndromes. It is due to changes in a single gene; BLM gene. The RecQ family DNA helicases Bloom syndrome protein (BLM) plays a key role in protecting the genome against deleterious changes. The RECQ helicase DNA is metabolically involved in many functions and is therefore related to the origins of common cellular phenotypes that are identified and defined for RECQ deficient cells. Pathologic mutations are involved in Bloom syndrome. BLM interacts with telomeric proteins and functions in telomere maintenance. Oxidative stress is ROS-induced oxidative injury which causes mitochondrial alternations and change cellular metabolism, the ratio of GSSG/GSH is considerably lowered in BS patient that makes them sensitive to oxidative stress as well as increase in ROS production. BS as compared to other diseases is caused by oxidative stress which is characterized by accumulation of cancer cells and degeneration of neurological cells. Many studies related to phenotype of BS have been done which not only explain its causes but also therapeutic strategies to combat this disease.
\end{abstract}

Volume 2 Issue 4 - 2017

\author{
Sikander Ali, Zainab Masood, Nimra Khan \\ Department of Molecular Biology, Government College \\ University, Pakistan
}

Correspondence: Sikander Ali, IIB, Government College University, Pakistan, Email zainabrajput196@gmail.com

Received: May 16, 2017 | Published: June 16, 2017

\section{Introduction}

Bloom $^{1}$ identified Bloom Syndrome for the very first time, recognized patients with skin changes, stature of short body and a particular butterfly rash across the sides of nose and cheeks that may also extend to hands and forearms. This rash particularly form when skin is exposed to sunlight and it may leads to hyperpigmentation and skin hyper. It is a pleiotropic genetic disorder with the prevalence of 1 per 40,000-100,000. It belongs to a group of chromosomal breakage syndromes (CBS).the characteristics of such and is an increased rate of chromosomal rearrangements and genomic instability either spontaneous or due to some sort of exposure to different DNAdisrupting factors such as external radiomimetic chemicals or ultraviolet (UV) light and ionizing radiation (IR). Bloom Syndrome is a very rare disease commonly associated with Jewish people mostly the Ashkenazi Jews approximately $1 / 3$ rd of the BS population. This explains why Jews are more familiar with this condition compared to the others. This type of disorder is genetic and most of the people it from their parents though they do not manifest any kind of signs and symptoms. $^{2,3}$

These people have possess low cell mediation and humoral immunity, and have greater chances of otitis media, pneumonia and diabetes mellitus as shown in Figure 1. The fertilization in BS patients is minimum or even negligible that is males are most probably infertile in this case, on the other side females are hypo fertile, this means that they have chances to fertile a normal off spring. Such patients have greater chances of having predeposition of cancerous cells in their body as well as these patients are might be suffering from diabetes mellitus. These patients are more susceptible to such genetic disorders as compared to others as shown in Figure 2. Common adult epithelial tumors such as colon, breast and lung cancer; leukemias and lymphomas; sarcomas; and rare pediatric tumors such as Wilms' tumors develop in these people. Cancer causes most of causalities in BS patients. ${ }^{4}$

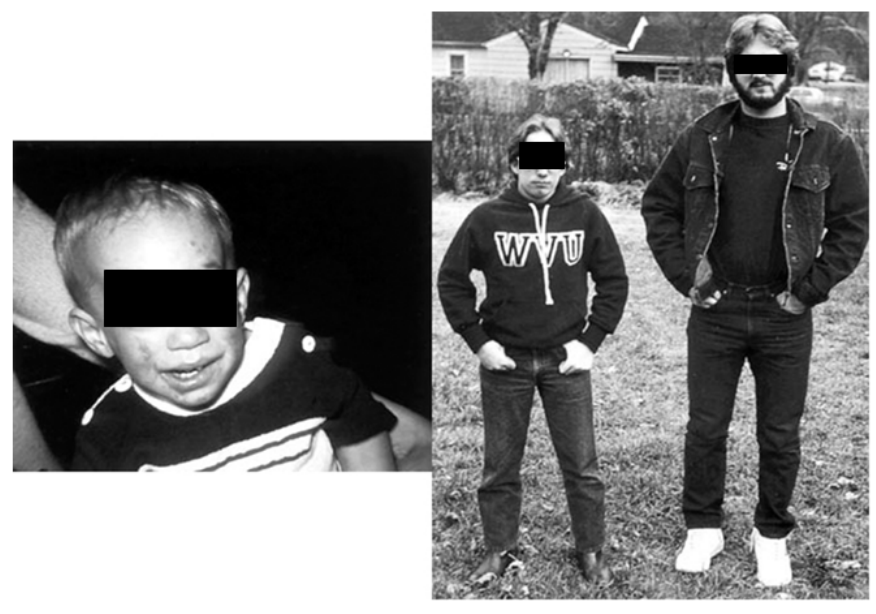

Figure I Registered patient of bloom syndrome with short body stature.

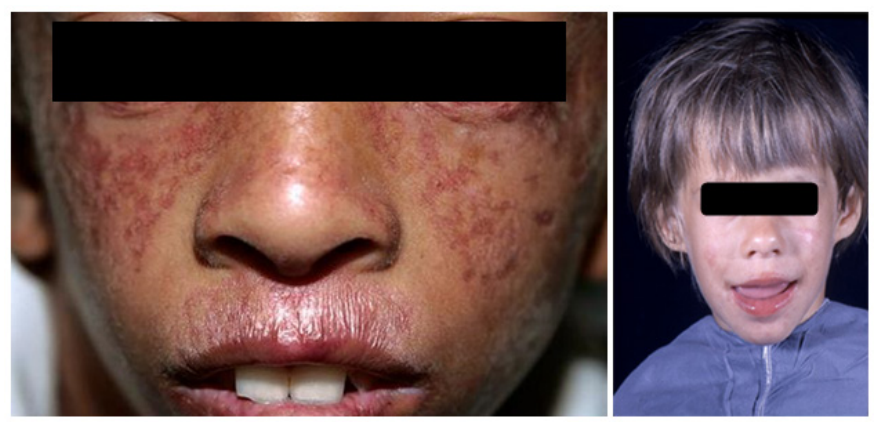

Figure 2 Sun burns visible in a BS patient and microcephaly.

\section{Genetics}

The most uncommon case BS (Bloom-Torre- Machacek syndrome) is due to disruption in a single gene; BLM (604610) which is present 
on 15q26.1. ${ }^{5}$ It encodes a DNA helicase RecQ protein that is vital for maintaining DNA stability by the processed involved in replication. The cell samples observed in BS patients show symptoms of DNA injury the chromatin material and cytoskeleton reorganization cells obtained from BS patients show signs of DNA injury, cytoskeleton abnormal cell cycle check points and extra amount of apoptotic cells production and even greater amount of cell death. Since it is an autosomal recessive syndrome so the BLM gene is inherited from parents and the chromosomes of progeny undergo some changes that is they change from one form to another as depicted in Figure $3 .^{6}$

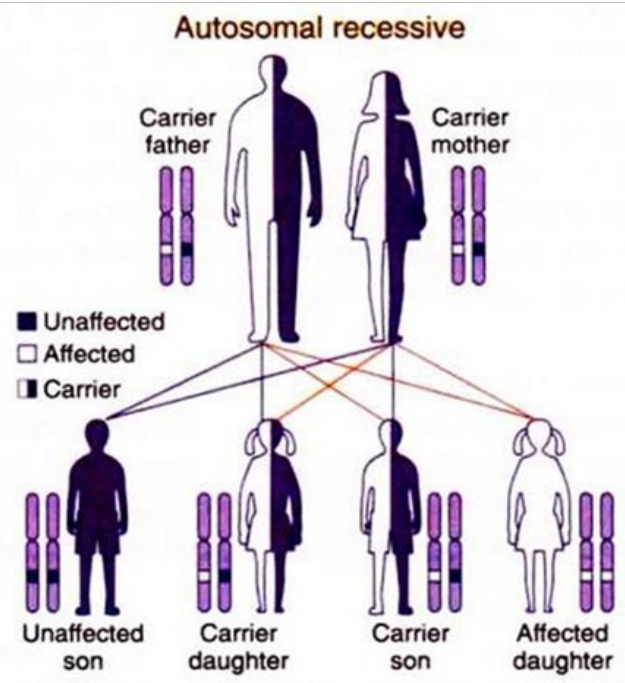

Relationship between two carrier parents and probabilities of children being unaffected, carriers, or affected

Figure 3 Normal and Effected progeny.

\section{Genomic instability and clinical features}

There is an increased frequency of sister chromatid exchanges which causes genomic instability on a molecular level in a cell of a BS patient so there is an increased risk of mutations in these cells more over many abnormalities can be seen in the processes like DNA synthesis, replication process in which abnormal replication intermediates start accumulating. Loss of BLM function on molecular level is crucial for genome instability and is linked to cancer predisposition. ${ }^{7}$ Patients with BS are characterized by small skull size (microcephaly strong growth deficiency they show Growth retardation with short body stature i.e. growth failure, Photosensitivity i.e. sunlight sensitivity they develop skin lesions on face after sunexposure, a variable immuno deficiency, neurological defects which are basically progressive cerebral degeneration, reduced fertility, premature aging, characteristic telangiectasia, increased cancer incidence and susceptibility i.e. a predisposition to a wide spectrum of cancers are amongst the major clinical complications that arise in the BS patients. Aberrant DNA damage response or genomic instability can explain these abnormalities. The RecQ family DNA helicases Bloom syndrome protein (BLM) plays a key role in protecting the genome against deleterious changes. BLM has distinct functions in the cells and all these clinical features observed in a BS patient suggest that the functional loss of BLM cannot be compensated for by the presence of the other protein (or of other RecQ members. Any mutations in these proteins lead to rare genetic diseases associated with cancer predisposition and accelerated aging which is premature aging in case of humans. It has been suggested that BS exist in a state of permanent oxidative stress. With respect to radiation sensitivity, the cells in a BS patients show pleiotropic characteristics including chromosome rearrangements, abnormal S-phase cycle checkpoints in addition to it decreased homologous recombination. ${ }^{8}$

\section{Role of Recq helicases}

In humans there are 5 RECQ genes and any genomic instability in 3 of these 5 cause genetic syndromes which are hereditary in nature these syndromes are associated with cancer pre- deposition. RecQ-like helicases, five categories that are discovered in humans include: BLM, WRN, RECQL1, RECQL4, and RECQL5. These RecQ-like helicases are a highly specific family of proteins which are difficult for storing genome integrity. The integrity of genome that are associated with the cellular specificity of RecQ like helicase is maintained by mutation that takes place within three of five RecQ genes this leads to number of heretable diseases that consequently leads to cancer deposition. Mutation taking place at the BLM gene causes a rare disorder in human, named as Bloom syndrome (BS). ${ }^{9}$ The RecQ gene has a major role in the repair, signaling, DNA formation and maintenance of telomeres. The important function of BLM is to unwind the intermediate of DNA. BLM has a complicated activity process that must be controlled to avoid the recombination events that may leads to the damage of genomic stability.

Modifications brought after the process of translation of BLM make it more refine. Sub cellular localization, protein-protein interactions, and protein stability are being regulated by BLM phosphorylation, ubiquitination, and simulation. DNA repair mechanism must be proper and should be on time to prevent genome of being unstable that has a drastic affect on human health. This leads to pre deposition of cancerous cells and early aging process. ${ }^{7}$ One family of highly specific proteins difficult for error-free DNA damage repair is the homologs of the bacterial RecQ DNA helicase. ${ }^{10}$

\section{Origin of cellular phenotypes and proliferative defects}

Metabolically the RecQ helicase DNA participates in different functions and that's why they are involved in common phenotype at cellular levels that are recognized and are analyzed when there is deficiency in RecQ cells. They leads to proliferative defects such as DNA damage and genetic or genomic instability. ${ }^{7}$ In growth culture slow growth represents an elongated cell cycle period, decreased growth and even cell death and cell aging. The cell growth is suppressed if the cell is deficient in BLM gene. ${ }^{11}$ BS cells isolated from patients and normal cells deficient of BLM are vulnerable to DNA-damaging or replication- blocking agents (chemicals, radiation, and reactive oxygen species). Most of these agents also selectively kill cells defective in RECQL, RECQL4 and RECQL5. Functional roles of RECQ helicases in human cells is provided by these DNA damaging agents.

\section{Molecular insights of missense mutations in human helicase genes}

Many pathologic mutations within BLM cause premature translation termination and mRNA destabilization, which results in a complete absence of notable BLM protein levels. ${ }^{12}$ Within the helicase domain highly conserved residues also get mutated which cause the expression of BLM proptein which is actually a mutant. It has not any ability to unwind the helical structure of DNA and its called a catalytic null. BLM and its homologues get precipitated in a number of pathways so in order to protect the DNA damaging RecQ like 
helicase activity must be controlled. ${ }^{13}$ The genetic mutations observed in unicellular and multicellular organisms have given $b$ the important informations about the DNA repair disorders. These mutations have shown that biochemically affected proteins play their part in such type of mutation. ${ }^{4}$ Splicing mutations or mutations in promoter site that easily disturb the normal process of transcription and translation and put a great influence on gene control, or nonsense mutations that code for truncated proteins can give important information for genetic disorders. Moreover missense mutations which cause single amino acid substitution can provide a proper understanding to many mechanisms and molecular functions of a cellular protein for example a DNA metabolic enzyme. In many situations, separation-of-function mutants that demolish the function of one protein but not the other one can be used to dissect helicase enzyme mechanism or molecular requirements for pathway function In vivo. One class of missense mutations that can be used for understanding the metabolic function of helicases in cellular DNA metabolism deactivates DNA unwinding and exhibits a negative phenotype that is dominant in nature In vivo. A negative mutation in the helicase is very dominant and it inhibits the lysis of TP in presence of water and the process of unwinding of DNA. Increased expression of a helicase protein that is catalytically inactivated may have a few drawbacks one of which is that the expression is different as compared to the normal expression of a mutant allele like the one found in a heterozygous individual. ${ }^{14}$

If the mutant DNA helicase protein in its ATPase or DNA unwinding activity is expressed it binds stably to DNA and as a consequence of which a static protein-DNA complex is formed that stops the procedures like DNA formation, repair of damaged DNA and formation of mRNA from DNA along with these processes this dead helicase protein that actually mutant in nature should maintain its ability to interact with other proteins as well and by doing so it high jacks many important DNA repair factors. Another possibility are a large number of helicases which are homo or hetero-oligomers i.e. mixed mutant/normal helicase multimer that are either nonfunctional in their activity of unwinding the DNA or completely lose such ability. ${ }^{15}$ There are certain naturally occurring but diseasecausing mutations which are a challenge because of their phenotypic expressions. As a result of such missense mutations a non-functional helicase protein is produced which requires investigation on molecular level because of these mutation the biological pathways fail to operate properly for example missense mutations in human SF2 DNA helicases which belongs to the RecQ and family of Fe-S and the Twinkle mitochondrial DNA helicase is important because many genetic disorders that are related to mutations (including missense mutation) in the human $\mathrm{C} 10$ or $\mathrm{f} 2$ mitochondrial helicase gene. ${ }^{16}$

\section{Cellular regulation of BLM function}

BLM regulates many steps of DNA damage signaling, repair, and replication. Most of which require BLM helicase function to act on the secondary structures of DNA. Interestingly, BLM functions in two opposing pathways regulate the repair of any DNA damage and thus retain genome stability in Table 1. Thus BLM is a crucial for DNA damage repair by HR (Holliday junction), which occurs at DSBs in cell cycle events that are late S and G2 phases. HR during the cell cycle or at illegitimate substrates, for example a stalled replication fork, can cause chromosome translocations or deletions. BLM acts on same sort of substrates to initiate HR in most of the cases, while inhibiting HR in others, it thus regulates HR which indicates that BLM activity must be regulated according to the cell requirement. Modern technologies and studies have revealed many types of BLM regulation, most specifically through many types of PTMs (post translational modifications). These mechanisms thus check the stability localization and interactions (with other proteins) of BLM demonstrated in Figure 4. ${ }^{5}$

Table I Antioxidant and oxidant parameters in patients with BS

\begin{tabular}{|c|c|c|c|c|}
\hline Antioxidant/ Oxidant parameter & Parameter changes & Biological material & Method & Number of patients( $\mathbf{N})$ \\
\hline \multicolumn{5}{|l|}{ Lipophilic antioxidants } \\
\hline \multirow[b]{2}{*}{$\alpha$-tocopherol } & $\approx$ & Plasma & HPLC & 4 \\
\hline & $\approx$ & & HPLC & 4 \\
\hline$\gamma$-tocopherol & $\approx$ & Plasma & HPLC & 4 \\
\hline \multicolumn{5}{|l|}{ Other non-enzymatic antioxidants } \\
\hline Oxidised glutathione (GSSG) & $\downarrow$ & whole blood & HPLC & 4 \\
\hline GSSG: GSH & $\downarrow$ & whole blood & HPLC & 4 \\
\hline Ratio & $\downarrow$ & whole blood & HPLC & 4 \\
\hline Glyoxal (Glx) & $\approx$ & & HPLC & 4 \\
\hline Methylglyoxal & $\approx$ & Plasma & HPLC & 4 \\
\hline (MGlx) & $\approx$ & & HPLC & 4 \\
\hline \multirow{2}{*}{ Uric acid (UA) } & $\uparrow$ & Plasma & HPLC & 4 \\
\hline & $\uparrow$ & & HPLC & 4 \\
\hline \multirow{2}{*}{ Ascorbic acid(AA) } & $\approx$ & Plasma & HPLC & 4 \\
\hline & $\approx$ & & HPLC & 4 \\
\hline
\end{tabular}


Table Continued....

\begin{tabular}{lllll}
\hline Antioxidant/ Oxidant parameter & Parameter changes & Biological material & Method & Number of patients(N) \\
\hline Oxidative modifications products & & & & \\
8-hydroxy-2- deoxyguano- sine (8- & $\uparrow$ & Leukocytes & LC & 4 \\
OHdG) & $\uparrow$ & & LC & 4 \\
& $\uparrow$ & Urine & LC & 4 \\
\hline
\end{tabular}

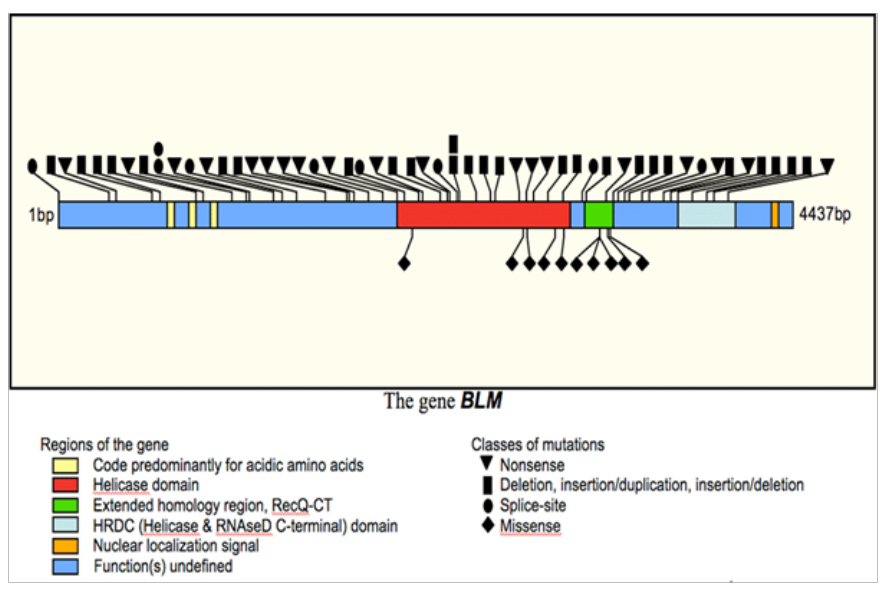

Figure 4 BLM gene.

\section{BLM function at telomeres}

The telomeres are present at the end of chromosomes, they are actually a complex of DNA and proteins and are involved in many crucial functions like maintenance of genomic integrity, has effects on the survival and division of cell. The length of telomeres is very crucial since it is involved in cell death by a process known as apoptosis and are known for their function in aging of the cell a phenomenon known as senescence in which the size of telomeres shortens during normal somatic cell division until and unless its ends are actively elongated. In the absence or inactivation of an enzyme specific to telomeres known as telomerase, cells use recombination and adopt a pathway called alternative lengthening of telomeres abbreviated as ALT- pathway in order to sustain its specific length.BLM binds with telomeric proteins and functions in telomere maintenance. The cells that lack BLM show more telomere defects, such as the loss of sister telomeres and production of telomere free ends of chromosomes and exhibit an increased telomere association between homologous chromosome arms. This situation is caused from the formation of unresolved replication intermediates or entangled telomeres. These telomere defects are responsible for premature aging problems in BS. ${ }^{17,18}$

\section{Pathophysiology and relationship with ROS}

A number of BS related pathologies like premature aging, growth retardation, insulin resistance, endocrine abnormalities and immuno deficiency may result from increased oxidative stress and oxidative damage. Oxidative stress is ROS-induced oxidative injury which causes mitochondrial alternations and change cellular metabolism in addition to gene expression, signal transduction pathways, cell differentiation and cell death by in BS patients depicted in Figure 5. In case of antioxidant defense, the formation and accumulation of oxidized products of the cellular DNA, proteins and lipids are responsible for the premature aging, neurodegeneration, carcinogenesis and chronic inflammation. ${ }^{18}$

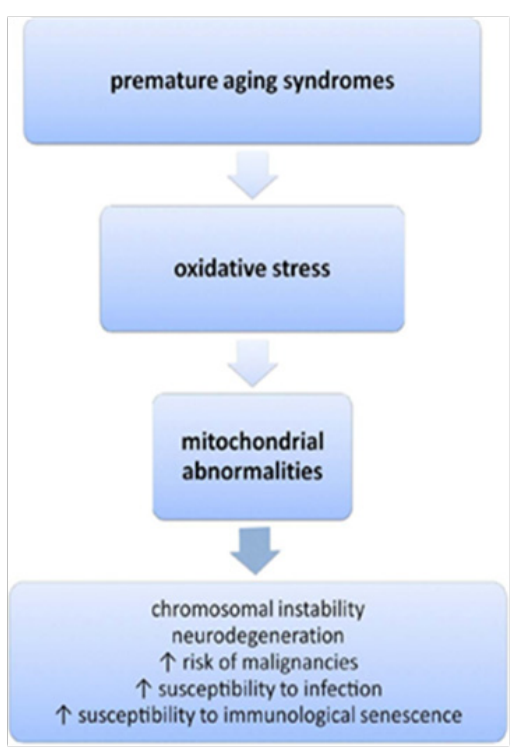

Figure 5 Premature aging and its relationship with oxidative stress.

Above information gave an idea about the oxidative modification products that are associated with the proper index of oxidative stress, therefore, oxidative stress is incorporated with bloom syndrome. The differences between oxidative stress and damages is due a minute number of subjects in conducted studies of date, this has no actual effect on original oxidant or antioxidant level in infected person. More research is needed in this regard, especially research concerning the examination of total oxidant or antioxidant capacity markers, individual enzymatic and non- enzymatic antioxidants as well as cellular oxidative modification products. Only the clear understanding of the oxidant or antioxidant equilibrium and mechanisms leading to oxidative stress and oxidative damage in BS cells will allow development of new, additional therapeutic proposals for patients suffering from these diseases. ${ }^{19}$

This syndrome is also known as premature ageing syndrome. It is characterized by instability of genome, central nervous system degeneration, cutaneous appendix dysfunction, malignancy risk as well as infection susceptibility and sensitive immune system (susceptible to more diseases). One of the major causes of this syndrome is constant oxidative stress provided by ROS endogenous overproduction as well as impairment of hemostasis taking place in mitochondria. The role of metabolism of oxygen is limited in the path physiological and clinical manipulation of this syndrome as well as in multiple complexities. The studies of pro-oxidants and antioxidants homeostasis gives the information about the intensity of disease and new or better meditation schemes provided to patients..$^{20}$ This phenomenon is very 
crucial. Oxidative stress associated with bloom syndrome is due to damage in DNA replication and other cycle abnormalities. Normal cells in bloom syndrome are less sensitive than the BS cells that is associated to the excessive production of endogenous ROS and prime important biomolecules such as proteins (leads to protein damage or oxidation), nucleic acids (leads to mutations in DNA), and lipids (leads to peroxidation in lipids). Oxidative stress leads to structural as well as functional changes to DNA, proteins and lipids. The most damage occurs is in DNA because such change leads to cellular mutations and consequently, cancer. Change in structure and function leads to mitochondrial dysfunction. Excessive production of ROS and oxidative stress induced in mitochondria causes much damage; this is also reported in BS patients and in modeled animals. ${ }^{21}$

Despite of their importance in tumor formation and early aging, BLM, gene responsible for bloom syndromeis a new target in cancer chemotherapy. Recent research has reported a new compound named as ML216 that is a particular inhibitor for BLM. This inhibitor inhibits the process of helicase in BLM 636-1298. Fragment of same structure was used that interpret the structural properties while competing with DNA binding site. Co-crystallization of BLM along with this inhibitor leads to a novel drug formation in future. Important drugs or medications are used to overcome the ROS production that plays important part in symptoms of this disease. It is associated with the molecular basis of bloom syndrome due to over-production of ROS in mitochondrial anomalies. Research has been done on the characteristics of ROS overproduction in DNA repair and maintenance. ${ }^{22}$

\section{Role of antioxidants}

No specificity has been demonstrated in selection of any nonenzymatic antioxidant. They might be glyoxal, methylglyoxal, ascorbic acid $\alpha$ - and $\gamma$ - tocopherol. Although, the levels of most important antioxidant in blood are urea and leukocyte, oxidative DNA damage marker -8 OHDG have been sigficantly demonstrated in BS patient as compared to healthy person and BS heterozygotes. ${ }^{23}$ However, the ratio of GSSG/GSH is considerably lowered in BS patient that makes them sensitive to oxidative stress as well as increase in ROS production. Increased levels of leukocytes and uric acid observed in BS patient during research conducted on bloom syndrome. A recent study is done on fibroblast line cells that explain the role of redox alterations in cells, in BS phenotype. This shows the increased levels of SOD activity and overproduction of ROS in BS cells. ${ }^{24}$

\section{Future prospects of antioxidants and strategies in BS}

Many studies related to phenotype of bloom syndrome have been done that not only explain the causes but also therapeutic strategies in this disease as compared to other disease whose major cause is oxidative stress are characterized by high accommodation of cancer causing cells and degeneration of neurological cells. Increased production of ROS in BS patients causes increased activity of antioxidant enzymes (CAT, GSH-Px and SOD) with a low concentration of another enzyme LWMA of low molecular weight. An efficient physiological response is required that minimal the effect of oxidative stress and its damage. Induction of an antioxidant defense system (AOS) is the most important process that limits the production of ROS that induces the oxidative stress as well as it regulates the ROS production that is linked with many other pathogenic and human diseases. If the oxidative stress then it causes depletion of antioxidants whether enzymatic and non-enzymatic. Thus, exogenous antioxidants are provided that are tempol, NAC, L-carnitine, iron, EUK-189 and CTMIO. Many studies that are done on this regard influence the use of antioxidant in treatment of oxidative stress, damage, neurological diseases and carcinogenic chemoprevention in modeled animals. ${ }^{25}$

L-carnitine is significant in treatment of BS syndrome as compared to mannitol that is a non-enzymatic inhibitor hydroxyl radical that is highly reactive. This is still under consideration that whether antioxidant or free radical inhibitor improves clinical condition and increase the survival time in a BS patient. Many antioxidants are also used in clinical studies. No research has been conducted on antioxidant treatment associated to lipophilic LWMA up till now. It shows that the antioxidant medications prove to be important in therapeutic strategy for such patients. Further studies related to this issue must be done on human models to improve the miserable conditions of such patients. ${ }^{26-33}$

\section{Conclusion}

The major phenotypic mark in bloom syndrome is chronic oxidative stress. The future directions totally understand the function of BLM and its molecular mechanism in this disease as well as its issues related to the health of humans. BS is occasional and is an autosomal disorder which is recessive in nature and is caused by heterozygous mutation in BLM that leads one individual to breast cancer and colorectal cancer. Similarly, the study of molecular damage in DNA molecule reveals the use of drugs in treatment of this disease as well as in minimizing development of cancer cells. Although, the cells isolated from the infected individuals show high genomic instabilities such as chromatid exchange and telomere shortening.

\section{Acknowledgements}

None.

\section{Conflict of interest}

Author declares that there is no conflict of interest.

\section{References}

1. Bloom D. Congenital telangiectatic erythema resembling lupus erythematosus in dwarfs: probably a syndrome entity. AMA Am J Dis Child. 1954;88(6):754-758.

2. Maciejczyk M, Mikoluc B, Pietrucha B, et al. Oxidative stress, mitochondrial abnormalities and antioxidant defense in Ataxiatelangiectasia, Bloom syndrome and Nijmegen breakage syndrome. Redox Biology. 2016.

3. Yates CC, Hebda P, Wells A. Skin wound healing and scarring: fetal wounds and regenerative restitution. Birth Defects Res Part C: Embryo Today. 2012;96(4):325-333.

4. Suhasini AN, Brosh RM. Disease-causing missense mutations in human DNA helicase disorders. Mutation Research/Reviews in Mutation Research. 2013;752(2):138-152.

5. Liu T, Huang J. DNA end resection: facts and mechanisms. Genomics proteomics \& bioinformatics. 2016;14(3):126-130.

6. Bugreev DV, X Yu, EH Egelman, et al. Novel pro- and antirecombination activities of the Bloom's syndrome helicase. Genes Dev. 2007;21(23):3085-3094.

7. Manthei KA, Keck JL. The BLM dissolve some in DNA replication and repair. Cell Mol Life Sci. 2013;70(21):4067-4084.

8. Bohm S Bernstein KA. The role of post-translational modifications in fine-tuning BLM helicase function during DNA repair. DNA repair (Amst). 2014;22:123-132. 
9. San FJ, Sung P, Klein H. Mechanism of eukaryotic homologous recombination. Annu Rev Biochem. 2008;77:229-257.

10. Guo R, Xuand D, Wang W. Identification and analysis of new proteins involved in the DNA damage response network of Fanconi anemia and Bloom syndrome. Methods. 2009;48(1):72-79.

11. Kitano K. Structural mechanisms of human RecQ helicases WRN and BLM. Front Genet. 2014;5:366.

12. Squadronemn S, Brizio P, Mancini C, et al. Blood metal levels and related antioxidant enzyme activities in patients with ataxia telangiectasia Neurobiol Dis. 2015;81:162-167.

13. Kee Y, A D'Andrea D. Expanded roles of the Fanconi anemia pathway in preserving genomic stability. Genes Dev. 2010;24(16):1680-1694.

14. Wallen RC, Antonellis A. To charge or not to charge: mechanistic insights into neuropathy-associated tRNA synthetase mutations. Current Opinion in Genetics \& Development. 2013;23:302-309.

15. German J, Crippa BD. Bloom's syndrome III. Chromosom. 1974:361-366.

16. Fagagna AD. Living On a Break: cellular senescence as a DNA-damage response. Nat Rev Cancer. 2008;8(7):512-522.

17. Harrigan JA, Bohr VA. Human diseases deficient in RecQ helicases. Biochimie. 2003;85(11):1185-1193.

18. Lillard-Wetherell. Association and regulation of the BLM helicase by the telomere proteins TRF1 and TRF2. Human Molecular Genetics. 2004

19. Campisi J. Aging, Cellular senescence and Cancer. Annu Rev of Physio 2013;75:685-705.

20. Wang W. Emergence of a DNA-damage response network consisting of Fanconianaemia and BRCA proteins. Nat Rev Genet 2007;8(10):735-748

21. Singleton MR, Dillingham MS, Wigley DB. Structure and mechanism of helicases and nucleic acid translocases. Annu Rev Biochem. 2007;76(1):23-50.
22. Symington LS, Gautier J. Double-strand break end resection and repair pathway choice. Annu Rev of Gen. 2011;45:247-271.

23. Dellaire G, Bazett-Jones DP. PML nuclear bodies: dynamic sensors of DNA damage and cellular stress. Bioessays. 2004;26(9):963-977.

24. Nguyen GH, Tang W, Robles AI. Regulation of gene expression by the BLM helicase correlates with the presence of G-quadruplex DNA motifs Proc Natl Acad Sci USA. 2014;111(27):9905-9910.

25. Monnat RJ. Human RECQ helicases: roles in DNA metabolism, mutagenesis and cancer biology. Seminars in cancer biology. 2010;20(5):329-339.

26. Chu WK, Hickson ID. RecQhelicases: multifunctional genome caretakers. Nat Rev Cancer. 2009;9(9):644-654.

27. Croteau DL, Popuri Opresko VA. Human RecQ Helicases in DNA Repair, Recombination, and Replication. Annual Rev Biochem. 2014;83:519-552.

28. Hand R, German J. A retarded rate of DNA chain growth in Bloom's syndrome. Proc Natl Acad Sci USA. 1975;72(2):758-762.

29. Hirschhorn R. In vivo reversion to normal of inherited mutations in humans. J Med Genet. 2003;40(10):721-728.

30. Huang S, Li B, Gray MD. The premature aging syndrome protein, WRN, is a $3^{\prime}$ to $5^{\prime}$ exonuclease. Nat Genet. 1998;20(2):114-116.

31. German J, Sanz Ciocci EN. Syndrome-causing mutations of the BLM gene. Hum Mutat. 2007;28(8):743-753.

32. German J. Bloom's syndrome. Cancer Genet Cytogenet. 1997:100-106.

33. Perrone S, Lotti F, Geronzi UE, et al. Oxidative stress in cancer-prone genetic diseases in pediatric age: the role of mitochondrial dysfunction Oxid Med Cell Longev. 2016:1-7.

34. Vindigni A, Marino F, Gileadi O. Probing the structural basis of RecQ helicase function. Biophys Chem. 2010;149(3):67-77. 